\title{
Timing and Frequency Synchronization Algorithm for Distributed MIMO- OFDM Systems
}

\author{
Jing Liu ${ }^{1}$, Jian Wang ${ }^{1}$, Kangli Zhang ${ }^{1}$, Jibo Wei ${ }^{1}$, Kui Liao ${ }^{2}$ \\ National University of Defense Technology ${ }^{1}$, Changsha, 410073, China \\ liujingaiseng@163.com \\ Automation Station of Yunnan Military Region ${ }^{2}$
}

\begin{abstract}
Keywords: OFDM; Distributed MIMO; Preamble; Time synchronization; Frequency synchronization
\end{abstract}

\begin{abstract}
In this paper, we investigate symbol timing offset and carrier frequency offset estimation problem in distributed MIMO-OFDM systems. In distributed MIMO-OFDM systems, signals arrive at the receiving antennas on different timings and are characterized by distinct CFOs. This property makes synchronization an extremely challenging task as compared with the centralized ones. A training sequence which consists of two OFDM symbols having a reduplicate and conjugate structure is proposed to perform synchronization. Timing offsets are estimated using crosscorrelation type algorithm. The fractional frequency offset is estimated using the phase difference of the two duplicated training symbols, and the integral frequency offset is estimated by utilizing the opposite shifts feature of cross correlation. The analysis and simulation results show that adopting the proposed preamble structure and synchronization algorithm, the receiver can estimate not only the STOs but also the CFOs from all transmitters. And the proposed algorithm is shown to have better performance, in terms of synchronization errors, than the UPSP method.
\end{abstract}

\section{INTRODUCTION}

Orthogonal frequency division multiplexing (OFDM) has become a prevalent technique for wireless communication, because of its high spectrum efficiency and robustness against the frequency selective fading channels [1]. In the meantime, multiple-input multiple-output (MIMO) technology has been proven feasible to enhance the capacity and performance of a communication system [2]. Currently, the combination of MIMO signal processing with OFDM modulation is regarded as an efficient solution for improving the data rates of four generation (4G) wireless communication systems working in frequency selective fading channels. However, MIMO-OFDM system requires more accurate synchronization technology than SISO-OFDM system to achieve its potential advantages.

In fact, MIMO systems can be built up in a centralized or a distributed way. For centralized MIMO systems, multiple antennas of the transmitter and the receiver are closely placed. Therefore, the signals sent from different transmitting antennas arrive at the receiving antenna simultaneously. The antennas of the transmitter or the receiver are closely placed on the same device and also are driven by the same oscillator. As a result, there is just one single carrier frequency offset appeared among all transmitting-receiving links. However, in some other application scenarios, due to hardware restrictions or cost limitations, it is impossible to plant multiple antennas on a single device. Hence, the MIMO systems equipped with distributed antennas is a promising solution to acquire space diversity. Since the antennas are sparsely distributed in the space, transmitting signals arrive at the receiving antennas on distinct timing due to different propagation paths and are contaminated by CFOs. This characteristic makes synchronization even more difficult in the distributed MIMO systems than the centralized ones.

Plenty of research work has been proposed to solving the synchronization problems of the MIMO-OFDM systems [3]-[10]. However, most of the algorithms are utilized in the centralized MIMO-OFDM systems [3]-[5]. They assume that the STOs and the CFOs of all transmittingreceiving pairs are the same. Hence they are not available for the distributed MIMO-OFDM 
systems. Guo et al. [6] exploited a synchronization means using unequal period synchronization patterns (UPSP) to estimate the timing offsets for the distributed MIMO-OFDM system, but the frequency offsets are not concerned. After that, Cheng et al. [7] made use of the UPSP of [6] for timing synchronization and added another two training sequences to accomplish CFOs estimation. However, the accessorial training sequences significantly increase the overhead of MIMO-OFDM system. Lately, in distributed MIMO-OFDM systems, Wang et al. [8] adopted the partial constant amplitude zero auto-correlation (CAZAC) sequences to form a novel preamble structure. It separates the training sequences of different transmitting antennas in both time and frequency domain, yet the preambles are not common for the real systems.

In this paper, a novel training structure is designed and an efficient timing and frequency offset estimator is proposed for the distributed MIMO-OFDM system. In the proposed approach, the signals from different transmitting antennas are separated using the CAZAC sequences. The coarse timing synchronization is accomplished by finding the peak of the good cross-correlation between receive signal and the training sequences, and the fine timing synchronization is accomplished by compensating the shift of the cross-correlation generated by integral frequency offset (IFO). The fractional frequency offset (FFO) is estimated using the phase difference of the two duplicated training symbols. Finally, the IFO is estimated by utilizing the opposite shifts of the crosscorrelation produced by the conjugate preambles. The simulation results show that, compared with the estimator in [7], the proposed estimator has a significantly higher acquisition probability of the timing offset. In addition, it is remarkable that the proposed approach can simultaneously estimate the fractional and integral frequency offsets from all transmitters to the destination.

The rest of this paper is organized as follows. In Section II, there is a typical distributed MIMOOFDM system model and the corresponding synchronization problems. In Section III, we propose a new preamble design for synchronization in distributed MIMO OFDM systems, along with the proposed timing and frequency synchronization algorithm. The proposed method is analyzed and compared to the estimator in [7] by computer simulation in Section IV. Finally, some conclusion remarks are made in Section V.

\section{MIMO-OFDM SYSTEM MODEL}

In this section, we briefly describe a classical distributed MIMO-OFDM system, along with its timing and frequency synchronization problems. Suppose that we have $N_{T_{M}}$ transmitting antennas and $N_{R_{N}}$ receiving antennas sparsely distributed in space to form a multi-antenna array as showed in Fig. 1, where the wireless link between each transmitter and the receiver adopts OFDM modulation and is characterized by distinct propagation delay, $\mathrm{CFO}$, and channel effect. Accordingly, the time domain samples of the transmitted baseband OFDM symbol by the transmitting antenna $m$ can be expressed as

$$
x_{m}(n)=\frac{1}{\sqrt{N}} \sum_{k=0}^{N-1} X_{m}(k) \exp (j 2 \pi k n / N)
$$

After the sending signal passes the multi-path fading channel $h_{m, i}$, the baseband signal received by $i$ th receiving antenna can be modeled as

$$
r_{i}(n)=\sum_{m=0}^{N_{T}-1} e^{j(2 \pi / N) \varepsilon_{m, i}\left(n-d_{m, i}\right)} \sum_{l=0}^{L-1} h_{m, i}(l) x_{m}\left(n-d_{m, i}-l\right)+w_{i}(n)
$$

where the notations with subscript $m$ are related to the $m$-th transmitting antenna, let $h_{m, i}(l)(l=0, \ldots, L-1)$ be the channel response from $m$ th transmitting antenna to $i$ th receiving antenna, where $L$ is the channel order. $d_{m, i}$ denotes the propagation delay normalized to the sampling period, $\varepsilon_{m, i}$ denotes the CFO normalized to the OFDM subcarrier spacing, $N$ is the fast Fourier transform (FFT) size of OFDM system. $\varepsilon_{m, i}=\varepsilon_{m, i}^{f}+\varepsilon_{m, i}^{i}, \varepsilon_{m, i}^{f}$ is the fractional frequency offset $\left(\varepsilon_{m, i}^{f} \in[-0.5,0.5]\right)$, $\varepsilon_{m, i}^{i}$ is the integral frequency offset. $w_{i}(n)$ is the noise contribution which is modeled as a circularly symmetric white Gaussian process with variance $\sigma_{w_{i}}^{2}=N_{0}$. 


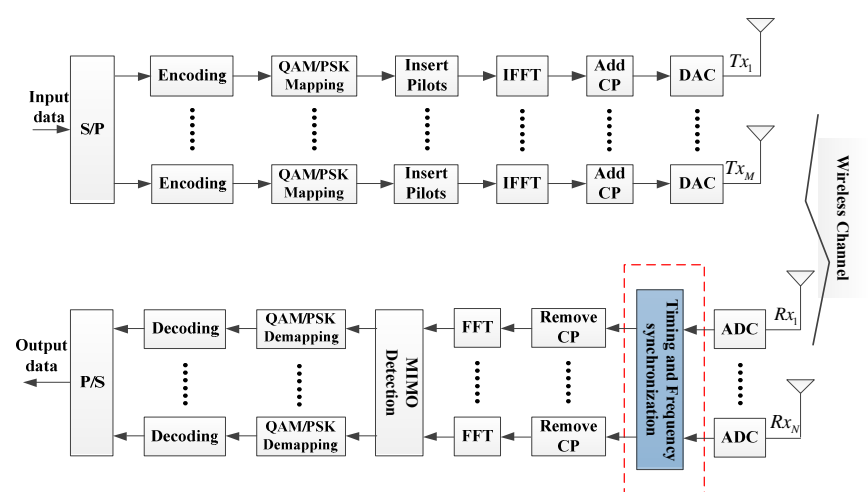

Fig. 1. A distributed MIMO OFDM system model.

Research has shown that if the symbol timing offset (STO) and CFO are not compensated, the orthogonality among the subcarriers will be destroyed by inter-symbol interference (ISI) and additional inter-carrier interference (ICI). Moreover, for MIMO-OFDM systems, there exists multiantenna interference (MAI) between the received signals from different transmit antennas. The STO, CFO and MAI will seriously deteriorate the performance of the MIMO-OFDM system. From the perspective of physical layer design, proper synchronization algorithms are crucial to build a successful product. Receivers can feed the estimating results back to the transmitters and ask them to adjust or use some other methods to recover the signals [10].

\section{THE PROPOSED APPROACH}

Synchronization algorithms for OFDM systems cannot be directly applied in distributed MIMOOFDM systems, because there are multiple timing offsets and CFOs. Therefore, the preamble structure and the synchronization algorithm must be redesigned.

\section{A. Proposed Training Preamble}

A good synchronization sequence structure must be such that, 1 . The time domain periodic autocorrelation of the sequence from all the transmitting antennas is an impulse function. 2. The preambles on the different transmitting antennas should be orthogonal and shift-orthogonal, for at least the channel length. 3 . The sequences can be directly tunable. 4 . The Fourier transform of the sequences should have a constant magnitude [4].

CAZAC sequences which satisfy the above conditions, are used to form the training sequence structure. As a contrast, the preamble structure of UPSP algorithm is showed in Fig. 2. The synchronization sequence is constituted by several repetitions of a shorter synchronization pattern. The idea of UPSP is to construct the synchronization sequence $L_{s s}$ for various transmitter antennas by assigning different values to $L_{s p}$.

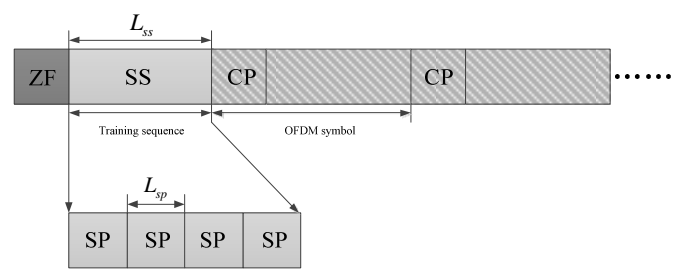

Fig. 2. Preamble structure for the UPSP algorithm.

The proposed time-domain preamble design is depicted in Fig. 3. The per-antenna preamble has two training symbols. The first training symbol is formed by a repetition of the CAZAC sequences. The second symbol is the conjugate sequence of the first one. The synchronization sequences $c_{1}$ transmitted from the first antenna are

$$
c_{1}(k)=e^{\left(j 2 \pi r k^{2} / N\right)}
$$

where $k \in[0, N / 2-1], r=N / 2-1$. The synchronization sequences $c_{m}$ with a cyclic shift applied to $s_{m}$ for the $m$-th antenna are

$$
C_{m}(k)=c_{1}\left[\left(k+s_{m}\right) \bmod N / 2\right]
$$


where $s_{m}=m N / N_{T}$.

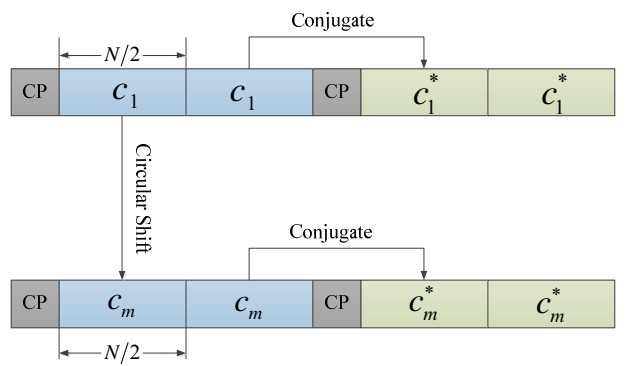

Fig. 3. Preamble structure for the proposed algorithm.

Based on the proposed preamble structure, synchronization is accomplished through timing synchronization, integer frequency offset estimation, and fractional frequency offset estimation.

\section{B. Timing Synchronization}

Inherently, the sharper timing-metric is, the less likely that the peak of timing-metrics is affected by neighboring values. Based on the proposed preamble design in Fig. 3, the timing estimation $\hat{d}_{m, i}$ associated with the $m$ th distributed transmitter can be determined by using typical correlation-based synchronization techniques. Hence, the timing metric is

$$
\Lambda_{m, i}(d)=\left[\sum_{n=0}^{N_{1}-1} r_{i}^{*}(d+n) c_{m}(n)\right] \cdot\left[\sum_{n=0}^{N_{1}-1} r_{i}^{*}\left(d+n+N_{1}\right) c_{m}(n)\right]
$$

where $r_{i}(d+n)$ shows the $d+n$ signal sampling in $i$ th receive antenna, $c_{m}(n)$ is CAZAC sequence in $m$ th transmit antenna, $N_{1}=N / 2$. Therefore, the timing offset estimation is

$$
\hat{d}_{m, i}=\underset{d}{\arg \max } \Lambda_{m, i}(d)
$$

\section{Frequency Synchronization}

1) Integer frequency offset estimation

Based on the proposed preamble design in Fig. 3, we analyse the influence of the IFO $\varepsilon_{m, i}^{i}$ on sequence $c_{m}$. Regardless of the effect of multi-path channel and noise, sequence $c_{m}$ is only under the influence of the IFO $\varepsilon_{m, i}^{i}$. The result is such that

$$
\begin{aligned}
c_{m}^{\prime}(k) & =c_{m}(k) e^{\left(j 2 \pi \varepsilon_{m, k}^{i} k / N\right)}=e^{\left(j 2 \pi r k^{2} / N\right)} e^{\left(j 2 \pi \varepsilon_{m, i}^{i} / N\right)}=e^{\left(j 2 \pi r k^{2} / N\right)} e^{-j 2 \pi k \varepsilon_{m, i}^{i} / N} \\
& =A e^{j 2 \pi r k^{2} / N} e^{-j 2 \pi r k \varepsilon_{m, i}^{i} / N} e^{j \pi r \varepsilon_{m, i}^{i} / 2 N}=A c_{i}\left(k-\varepsilon_{m, i}^{i} / 2\right)
\end{aligned}
$$

where $A=e^{-j \pi(N-1) \varepsilon_{m, i}^{i} / 2 N}$ has nothing to do with $k$. According to (7), the IFO $\varepsilon_{m, i}^{i}$ make sequence $c_{m}$ rotate left $\varepsilon_{m, i}^{i} / 2$. In a similar way, $\varepsilon_{m, i}^{i}$ make sequence $c_{m}^{*}$ rotate right $\varepsilon_{m, i}^{i} / 2$. IFO estimation $\hat{\varepsilon}_{m, i}^{i}$ is accomplished by exactly using above characteristic of CAZAC sequence which is shown in Fig. 4.

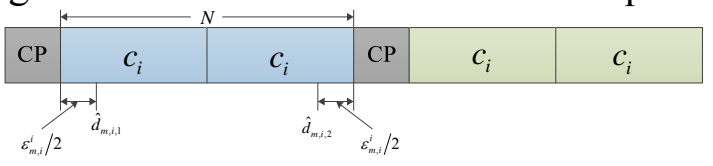

Fig. 4. The influence of integer frequency offset.

Thus, we define the complex correlations $\Lambda_{m, i, 1}, \Lambda_{m, i, 2}$ between signal sampling $r_{i}$ in $i$ th receive antenna and sequences $c_{m}, c_{m}^{*}$ respectively as

$$
\begin{aligned}
& \Lambda_{m, i, 1}(d)=\left[\sum_{n=0}^{N_{1}-1} r_{i}^{*}(d+n) c_{m}(n)\right] \cdot\left[\sum_{n=0}^{N_{1}-1} r_{i}^{*}\left(d+n+N_{1}\right) c_{m}(n)\right] \\
& \Lambda_{m, i, 2}(d)=\left[\sum_{n=0}^{N_{1}-1} r_{i}^{*}(d+n) c_{m}^{*}(n)\right] \cdot\left[\sum_{n=0}^{N_{1}-1} r_{i}^{*}\left(d+n+N_{1}\right) c_{m}^{*}(n)\right]
\end{aligned}
$$

Subsequently, the timing offset estimations $\hat{d}_{m, i, 1}, \hat{d}_{m, i, 2}$ getting from equation (8) and (9) respectively are

$$
\hat{D}=\underset{d}{\arg \max }\left\{\begin{aligned}
\Lambda_{m, i, 1}(d) & \Rightarrow \hat{d}_{m, i, 1} \\
\Lambda_{m, i, 2}(d) & \Rightarrow \hat{d}_{m, i, 2}
\end{aligned}\right.
$$


As we can see from Fig 4, $\varepsilon_{m, i}^{i}$ make the estimations $\hat{d}_{m, i, 1}, \hat{d}_{m, i, 2}$ respectively rotate right and left $\varepsilon_{m, i}^{i} / 2$. Finally, IFO estimation $\hat{\varepsilon}_{m, i}^{i}$ is given by

$$
\hat{\varepsilon}_{m, i}^{i}=N-\left(\hat{d}_{m, i, 2}-\hat{d}_{m, i, 1}\right)
$$

Once the IFO estimation $\hat{\varepsilon}_{m, i}^{i}$ is obtained, real time synchronization $\hat{d}_{m, i \text {,real }}$ can be performed by correcting initial timing offset estimation $\hat{d}_{m, i}$ with $\hat{\varepsilon}_{m, i}^{i}$. Hence, the real timing offset estimation is

2) Fractional frequency offset estimation

$$
\hat{d}_{m, i, \text { real }}=\hat{d}_{m, i, 1}-\hat{\varepsilon}_{m, i}^{i} / 2=\hat{d}_{m, i, 2}+\hat{\varepsilon}_{m, i}^{i} / 2-N
$$

FFO $\hat{\varepsilon}_{m, i}^{f}$ is mainly caused by the difference in oscillator frequencies at the transmitters and the receiver. We should estimate this frequency offset and compensate the received signals for it. The FFO can be estimated using the phase of the complex correlation $\Lambda_{m, i}$

$$
\hat{\varepsilon}_{m, i}^{f}=-\frac{1}{\pi} \arg \left[\Lambda_{m, i, 1}\left(d_{m, i, \text { real }}\right)+\Lambda_{m, i, 2}\left(d_{m, i, \text { real }}\right)\right]
$$

\section{SIMULATION RESULTS}

In this section, the performance of the proposed scheme is demonstrated and analyzed. The performance of the estimator in [7] will also be studied and used as a benchmark. For the sake of brevity, distributed MIMO-OFDM systems with two distributed transmitting antennas and one receiving antenna are simulated, where each packet is composed of a preamble followed by random OFDM data symbols. The main simulation parameters are based on summarized in Table I. AWGN and ITU Vehicle-A [12] (multi-path channel) have been used to simulate in the channel propagation. Assume that every wireless signal passes through an independent wireless channel. And the channel is assumed to be quasi-stationary during one OFDM symbol length. The CFOs are $\varepsilon_{1,1}=20.25$, $\varepsilon_{1,1}^{i}=20, \varepsilon_{1,1}^{f}=0.25, \varepsilon_{2,1}=40.15, \varepsilon_{2,1}^{i}=40, \varepsilon_{2,1}^{f}=0.15$. In the following, all the results are averaged over 1000 Monte Carlo realizations.

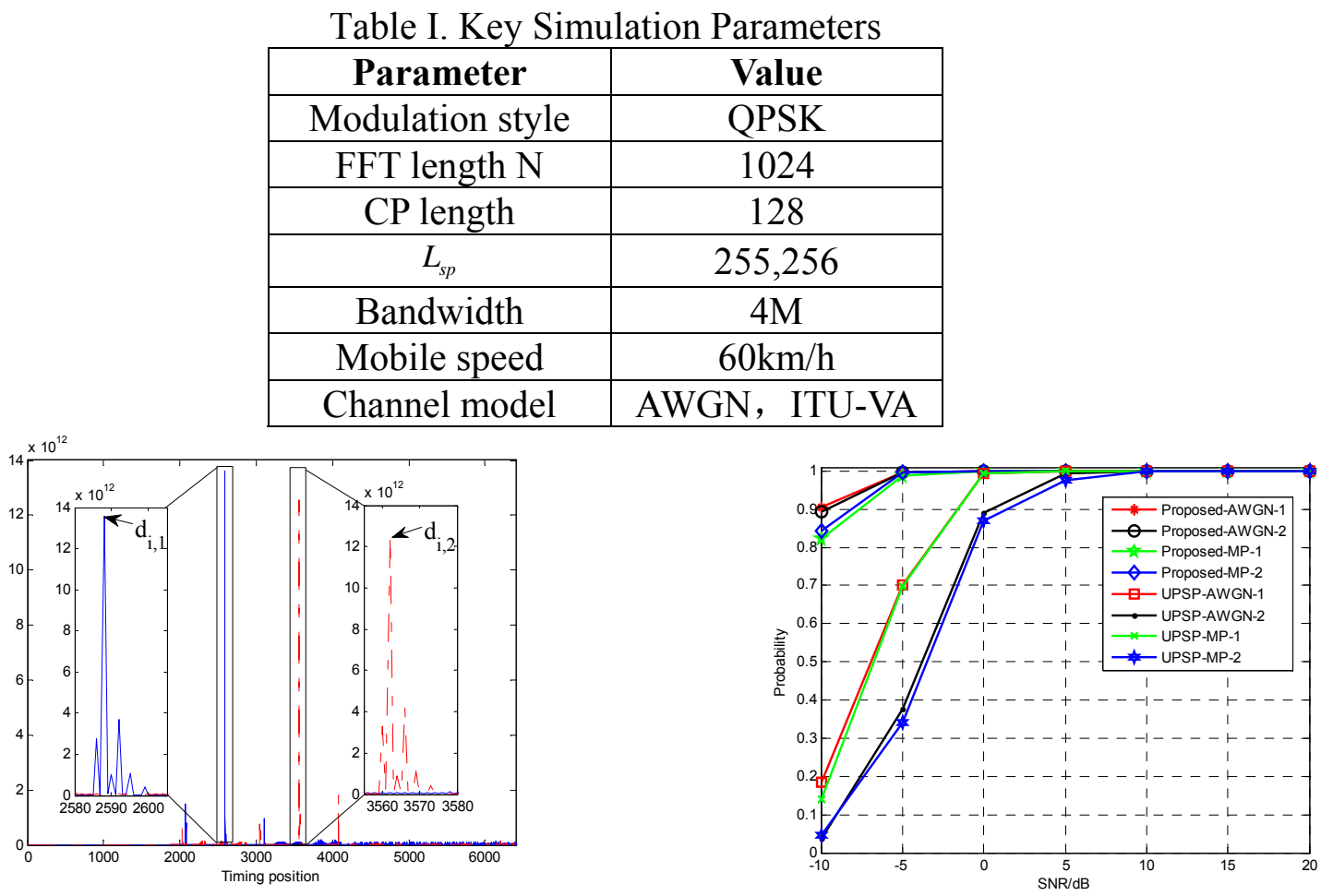

Fig. 5. Timing metric curve in Vehicular-A channel Fig. 6. Probability of timing offset estimation

CAZAC sequences have a good cross correlation and auto-correlation characteristics. From Fig. 5 , we can see that proposed method has a sharp metric function under multi-path channel. Although in multi-path channel the metric has several peaks, the peak of main path is much higher than others. 
And this contributes to improve the performance of timing synchronization. In Fig. 6, we demonstrate the proposed method performance in terms of the probability of successful timing acquisition in AWGN and multi-path channels. It can be observed that the proposed scheme outperforms the UPSP method especially in multi-path channel.

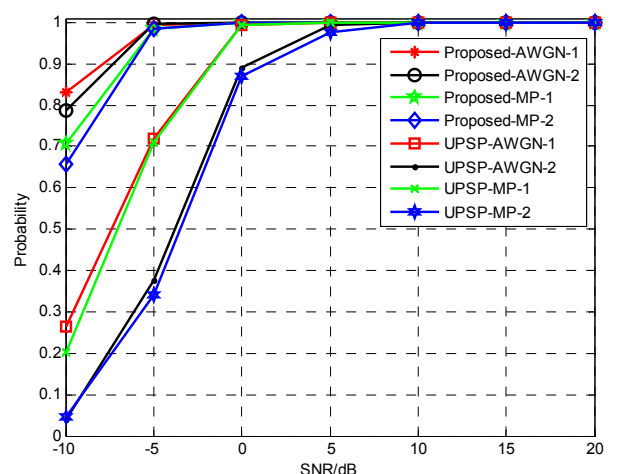

Fig. 7. Probability of integer frequency offset estimation offset estimation

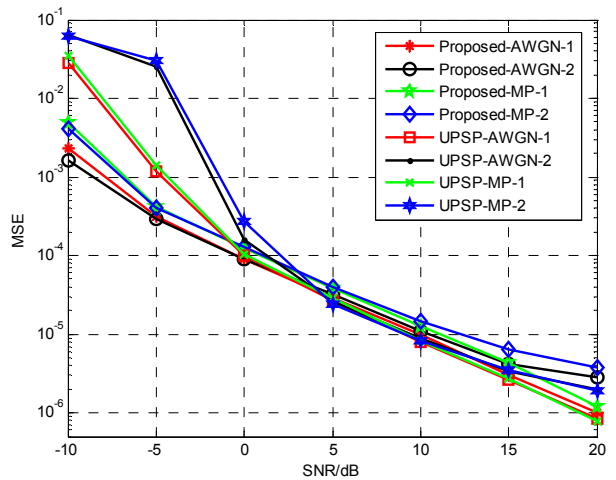

Fig. 8. MSE of fractional frequency

IFO acquisition probability, demonstrated in Fig. 7, shows that the proposed method performs better than the UPSP method in both AWGN and ITU Vehicular-A channels. The UPSP algorithm does not consider the shift characteristics of the CAZAC sequences in the time domain. Therefore, IFO will lead to the wrong timing offset estimation. Finally, IFO estimation will be not correct. In the simulations, we assume that the timing offset estimation has been corrected for the UPSP. From Fig. 7, we can obtain that at high SNR, the probability of the proposed algorithm is close to 1; in low SNR, it is much higher than that of UPSP algorithm.

Fig. 8 shows the MSE of FFO estimations in AWGN channel and ITU vehicular-A channel. As we can see from Fig. 8, when the SNR is less than 0dB, the MSE of proposed method is less than the UPSP algorithm. When the SNR is greater than $5 \mathrm{~dB}$, the MSE of proposed method is slightly larger than the MSE of UPSP algorithm. Both of the MSE are lower than $10^{-4}$. And compared to the performance under AWGN channel, the performance under multi-path channel is not significantly deteriorated. UPSP algorithm accomplish frequency offset estimation after FFT operation, therefore the complexity of UPSP algorithm is higher.

\section{v. CONCLUSIONS}

This paper proposed and analyzed an efficient and accurate time and frequency synchronization for distributed MIMO-OFDM system. Based on the CAZAC sequences, the proposed synchronization sequences can be utilized to estimate the different time offsets and different CFOs. In comparison to the conventional synchronization method using UPSP as in [7], it has been demonstrated by simulations that this method provides an efficient approach for timing and frequency synchronization for a distributed MIMO-OFDM system.

\section{REFERENCES}

[1] Schmidl T M, Cox D C. Robust frequency and timing synchronization for OFDM[J]. Communications, IEEE Transactions on, 1997, 45(12): 1613-1621.

[2] Paulraj A J, Gore D A, Nabar R U, et al. An overview of MIMO communications-a key to gigabit wireless[J]. Proceedings of the IEEE, 2004, 92(2): 198-218.

[3] Stuber G L, Barry J R, Mclaughlin S W, et al. Broadband MIMO-OFDM wireless communications[J]. Proceedings of the IEEE, 2004, 92(2): 271-294.

[4] Mody A N, Stuber G L. Synchronization for MIMO OFDM systems[C]//Global Telecommunications Conference, 2001. GLOBECOM'01. IEEE. IEEE, 2001: 509-513. 
[5] Van Zelst V, Schenk T C W. Implementation of a MIMO OFDM-based wireless LAN system[J]. Signal Processing, IEEE Transactions on, 2004, 52(2): 483-494.

[6] Guo F, Li D, Yang H, et al. A novel timing synchronization method for distributed MIMOOFDM system[C]//Vehicular Technology Conference, 2006. VTC 2006-Spring. IEEE 63rd. IEEE, 2006: 1933-1936.

[7] Cheng Y, Jiang Y, You X. Preamble design and synchronization algorithm for cooperative relay systems[C]//Vehicular Technology Conference Fall (VTC 2009-Fall), 2009 IEEE 70th. IEEE, 2009: $1-5$.

[8] Wang C L, Wang H C, Chen Y Y. A synchronization scheme based on partial Zadoff-Chu sequences for cooperative MIMO OFDM systems[C]//Wireless Communications and Networking Conference (WCNC), 2012 IEEE. IEEE, 2012: 925-929.

[9] Yang G, Wang C L, Wang H C, et al. A new synchronization scheme for OFDM-based cooperative relay systems[C]//Global Telecommunications Conference (GLOBECOM 2010), 2010 IEEE. IEEE, 2010: 1-5.

[10] Deng K. Frequency offset compensation for spatial multiplexing MIMO-OFDM systems with distributed transmit antennas[C]//nformation Science and Technology (ICIST), 2012 International Conference on. IEEE, 2012: 430-433.

[11] Chu D. Polyphase codes with good periodic correlation properties [J]. IEEE Transactions on information theory, 1972: 531-532.

[12] ITU-R M.1225, Guidelines for Evaluation of Radio Transmission Technologies for IMT$2000[S], 1997$. 